\title{
Neutronical aspects of a decay heat measurement: the PRESTO experiment
}

\author{
Francesco Muratori \\ Cadarache, DER, SPRC, LE2C \\ CEA, DEN \\ F-13108 Saint-Paul-lez-Durance, \\ France \\ francesco.muratori@cea.fr \\ Christophe Le Niliot \\ CNRS, IUSTI \\ Aix Marseille Univ \\ Marseille, France \\ christophe.leniliot@univ-amu.fr
}

\author{
Frédéric Nguyen \\ Cadarache, DER, SPRC, LE2C \\ CEA, DEN \\ F-13108 Saint-Paul-lez-Durance, \\ France \\ frederic.nguyen@cea.fr \\ Romain Eschbach \\ Cadarache, DER, SPRC, LE2C \\ CEA, DEN \\ F-13108 Saint-Paul-lez-Durance, \\ France \\ romain.eschbach@cea.fr
}

\author{
Christian Gonnier \\ Cadarache, DER, SRJH \\ CEA, DEN \\ F-13108 Saint-Paul-lez-Durance, \\ France \\ christian.gonnier@cea.fr
}

\begin{abstract}
Decay heat is the thermal power released by radioactive decays of unstable isotopes after the nuclear reactor shutdown, and delayed fission reactions. It constitutes a key parameter for the nuclear reactor safety and the nuclear fuel cycle; for this reason, design codes have to be qualified by comparison with experimental measurements. The CEA's package DARWIN2.3 has been qualified for the calculation of PWR decay heat with two integral measurements: the MERCI experience and the CLAB laboratory's experiments; performed respectively on the following cooling time intervals: $\mathbf{4 0} \mathrm{min}-\mathbf{4 0}$ days and 12 years -25 years. A lack of validation in the first hour of cooling time requires to consider large margins on the calculated decay heat value. As a result, delays in core unloading, intervention of human operators and safety systems dimensioning may occur. The PRESTO experiment, under conception at CEA, deals with a decay heat measurement between 1 and 40 minutes of cooling time for a PWR fuel sample irradiated in the Jules Horowitz Reactor (JHR). A previous thermal study showed that measurements could be sensitive to the decay heat 1 minute after the beginning of the cooling time. Now, a more precise estimation of power sources was performed with the Monte-Carlo code TRIPOLI. In this framework, four different device configurations were considered. Our results show that the irradiation power is not enough elevated in configurations where a tungsten shield is present.
\end{abstract}

\section{Keywords - JHR, PRESTO experiment, TRIPOLI.}

\section{INTRODUCTION}

Decay heat represents the thermal power released by radioactive decays of unstable isotopes after the nuclear reactor shutdown, and delayed fission reactions. Generally, radioactive decay energy is released in the form of beta (electrons) and gamma (photons) radiations; the formers are entirely absorbed in the nuclear fuel, whereas the latter both in the fuel and outside. If $N$ unstable isotopes are present, decay heat time-dependent value is calculated as the summation of the decay heat released by each isotope [1]

$$
P_{r}(\tau)=\sum_{i=1}^{N} E_{i} A_{i}(\tau)
$$

where $E_{i}[\mathrm{~J}]$ and $A_{i}\left[\mathrm{~s}^{-1}\right]$ stand respectively for the energy released and the activity, i.e. the number of decays per second of the i-th isotope. Decay heat increases with the fuel burnup until saturation levels of fission products concentrations are reached, and its contributors change according to the cooling time $(\tau)[2]$; as it is shown in [1], they correspond to delayed fissions and fission products in the first minute of cooling time, fission products between one hundred seconds and twenty years and heavy nuclides in the very long term.

The decay heat constitutes a key parameter for the design of nuclear reactor safety systems and the backend of the nuclear fuel cycle. For this reason, design codes have to be validated by comparison with experimental measurements. Nowadays, the CEA's package DARWIN2.3 is qualified in PWR conditions of irradiation with two integral decay heat measurements: the MERCI experiment [3] and the CLAB laboratory's experiments [4]; before, others qualifications were performed concerning the decay heat produced by elementary fission events of fissile materials [5]. The validations cover cooling time intervals going from 40 minutes to 40 days, for the MERCI experiment, and from 12 years to 25 years, for the CLAB's experiments. The lack of a validation in the very short cooling time (and the associated uncertainty), constrains the nuclear industries to take large margins on the calculated decay heat value. As a result, several issues in nuclear reactor safety occur with respect to thermal and radioactivity aspects (e.g. delays for core unloading, delays in the intervention of human operators, core cooling dimensioning in case of a Loss Of Coolant Accident, LOCA).

The PRESTO experiment [6] (PoweR Estimation for Short Time Optimization), under conception at CEA, deals with a decay heat measurement between 1 and 40 minutes of cooling time for a PWR fuel sample irradiated in the Jules Horowitz Reactor (JHR). Owing to the very short cooling times, the experiment must be performed in the reactor region, which brings on the measurement an important amount of perturbations due to the reactor core decay heat. In a precedent paper [6], we performed a sensitivity study of a determined device configuration by using a preliminary estimation of power sources, thanks to the feedback of SRJH (the CEA division in charge of JHR design), and a numerical scheme simulating heat transfers in the experimental device in 2D cylindrical geometry $(r, z)$. Results showed that measurements were not sensitive enough to estimate the decay heat fraction released out of the fuel sample. As a result, we need a better estimation of power sources and 3D heat transfer modeling considering the non-azimuthal symmetry of irradiation. Thus, we used the TRIPOLI Monte- 
Carlo code to estimate nuclear heating during irradiation, at $\tau=0$, and after the reactor shutdown, at $\tau=2$ min and $\tau=1 \mathrm{~h}$; simulations were performed by considering 4 different device configurations.

In this paper, we compare TRIPOLI results with design parameters: irradiation power, burn-up, irradiation time, required for the conception of the PRESTO experiment. In so doing, we see how the choice of the experimental device influences the experiment conception.

\section{THE PRESTO EXPERIMENT}

The PRESTO experiment is designed to estimate the decay heat released by a PWR fuel sample, with an aimed confidence interval of $99 \%$ for a representative PWR fuel burn-up, between 1 and 40 minutes of cooling time. In this framework, we have to reproduce conditions of irradiation of current PWRs, and keep irradiation times in the JHR no longer than one year.

\section{A. The Jules Horowitz Reactor (JHR) and the experiment development}

The Jules Horowitz Reactor [7] (JHR), under construction in the site of Cadarache, was chosen for performing the PRESTO experiment. This reactor is devoted to the study of

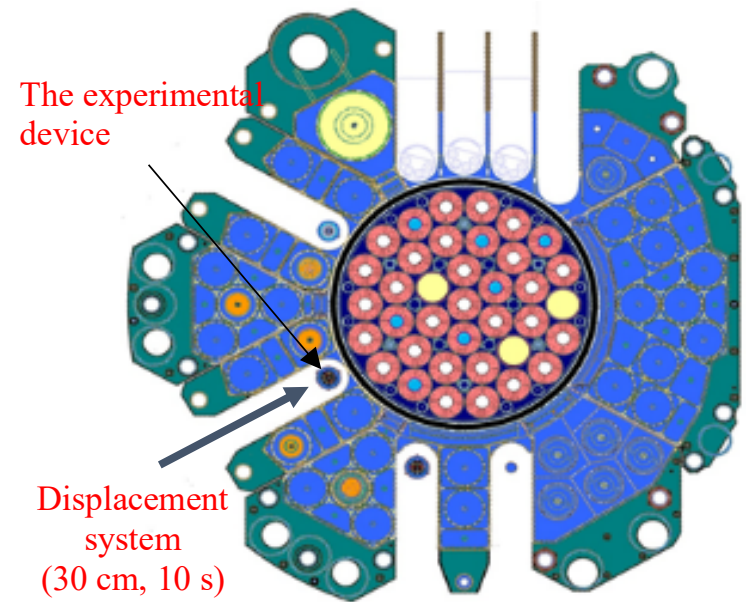

Fig. 1. The Jules Horowitz reactor core and reflector; the position of the experimental device: the JHR reflector on the displacement system.

materials and fuel behavior under irradiation as well as to medical isotopes production. The PRESTO experiment is designed to take place on the displacement system, situated in the JHR reflector (see figure 1); this one allows to master the irradiation power by regulating the distance between the experimental device and the reactor core.

The experiment is foreseen in a sequence of three phases: the irradiation, the stop of the irradiation and the measurement. At first, the experimental device is placed in the vicinity of the reactor core to perform the irradiation. When the desired fuel burn-up is achieved, the stop of the irradiation occurs by carrying out the reactor shutdown (SCRAM), the cooling phase starts, and the experimental device is moved back, by using the displacement system, to preserve it from perturbations coming from the reactor core. In this phase, it is important to remove thermal inertia stored in the device during the irradiation. At 1 minute of cooling time the measurement phase starts.

\section{B. The experimental device}

The experimental device scheme is reported in figure 2 . The geometry is the cylindrical one, the height and the diameter are respectively around $40 \mathrm{~cm}$ and $10 \mathrm{~cm}$. Decay heat power, released by the fuel sample, is deposited in the fuel and the surrounding environnement; particularly, the

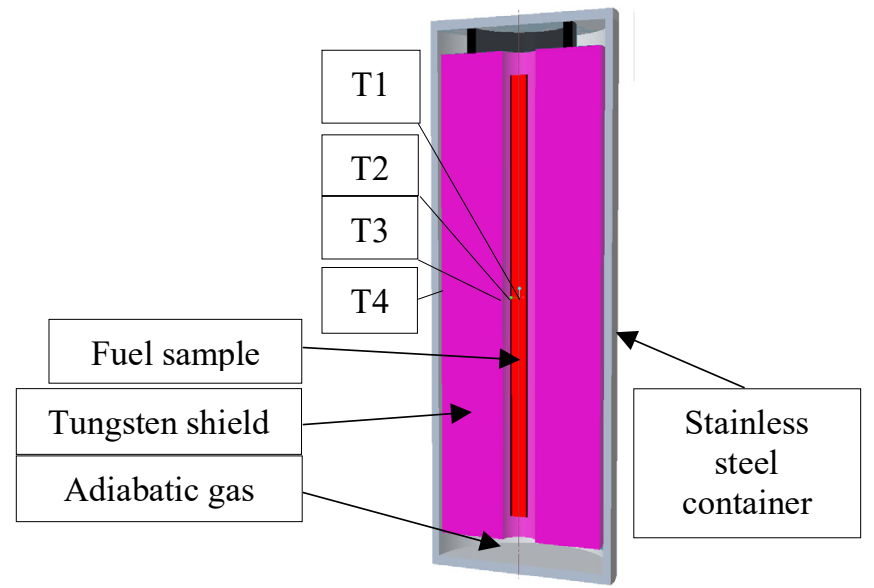

Fig. 2. The experimental device. In red: the fuel sample. In violet: the tungsten shield. In grey: the stainless steel container. T1, T2, T3, T4 are thermocouples placed in the fuel sample and the tungsten shield.

tungsten shield has been designed to collect the decay heat fraction leaving the fuel sample. The measurement principle consists in quantifying decay heat through the structures enthalpy instantaneous variation. In this way, the estimation parameter is the temperature temporal derivative of each structure; particularly we refer to the fuel sample and the tungsten shield. To do this, thermocouples have been located in the experimental device, as it is shown in figure 2 , to measure the temperature evolutions. For simplifying the device physics and thus its thermal modeling, heat transfers among structures must be limited. For this reason, an insulating gas is injected in the device chamber (see figure 2).

\section{The determination of decay heat}

As we are in the very short cooling times, temperature fields are highly unsteady. As a result, to determine decay heat starting from thermocouples measurements, we need for a heat transfer modeling of the experiment. In this way, the decay heat estimation is performed by means of an inverse technique [8]. Figure 3 shows the scheme for the decay heat estimation.

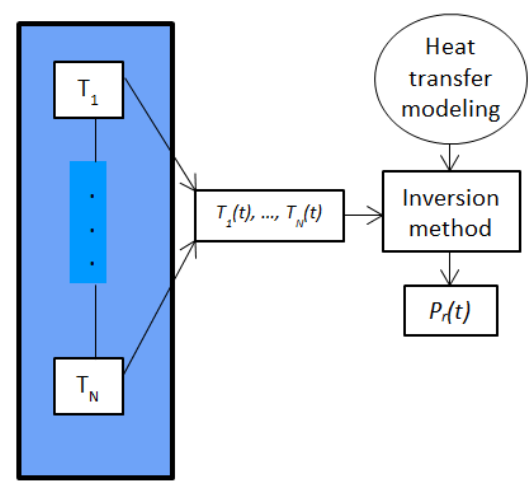

Fig. 3. Decay heat estimation. The $N$ thermocouples measure the temperature evolutions. These are used with a heat transfer modeling to estimate decay heat through the linear inversion method. 


\section{THE ESTIMATION OF NUCLEAR HEATING}

As our previous results showed that measurements were not sensitive enough to estimate the decay heat fraction released in the tungsten shield [6], we need for a more accurate sensitivity calculation to evaluate the feasibility of the PRESTO experiment. This involves a better estimation of nuclear heating and a more accurate modeling of heat transfers in the experimental device. To carry out the nuclear heating estimation we determined at first the power sources in the reactor core and the fuel sample through the evolution code MENDEL; results were then used as input in the Monte Carlo code TRIPOLI to evaluate the corresponding nuclear heating of the device.

\section{A. Simulation set-up}

Calculations were performed at three instants of cooling time: $\tau=0$ (i.e. the reactor shutdown), $\tau=2 \min$ and $\tau=1 \mathrm{~h}$, and by considering four different device configurations (see figure 3).

$\square$ Configuration 1: the fuel rod (at the center in red) is surrounded by one tungsten shield (in violet), the device is cooled with water (in blue) and enclosed in a stainless steel container (in claret-red).

$\square \quad$ Configuration 2: the fuel rod (at the center in red) is surrounded by two tungsten shields (in violet), which are separated by a stainless steel wall (in claret-red). The device is cooled with water (in blue).

$\square \quad$ Configuration 3: the fuel rod (at the center in red) is surrounded by an internal shield of tungsten (in violet) and an external shield of zirconium (in green), which are separated by a stainless steel wall (in claret-red). The device is cooled with water (in blue).
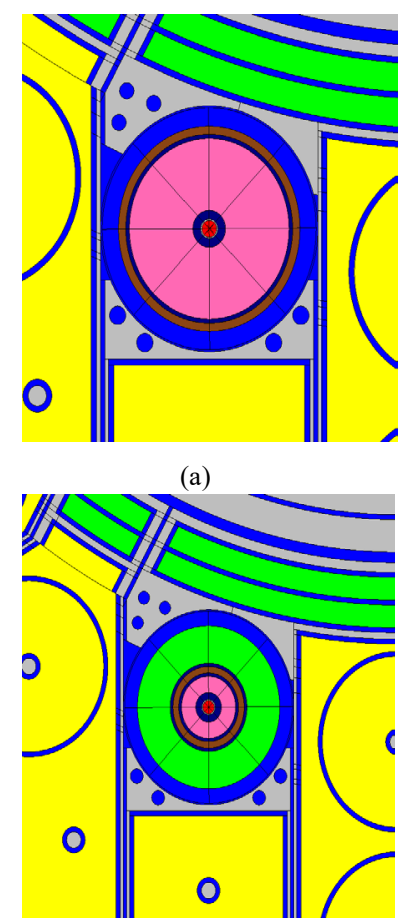

(c)

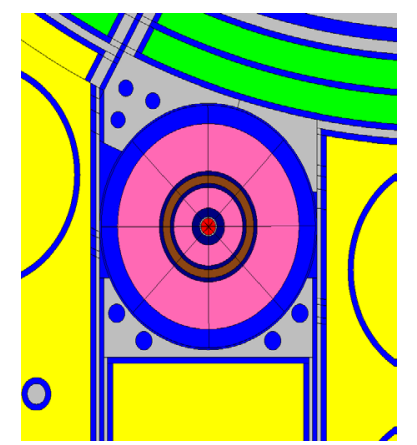

(b)

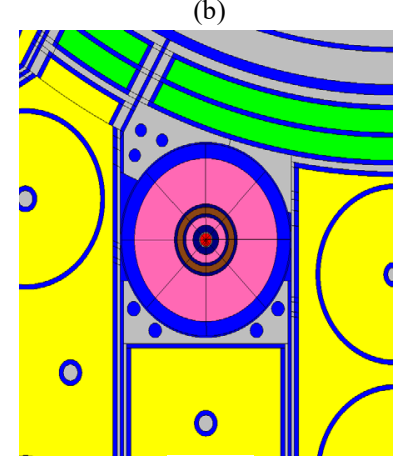

(d)

Fig. 4. Device configurations. (a): configuration 1. (b): configuration 2. (c): configuration 3. (d): configuration 4. 


\section{B. Results \\ 1) The irradiation phase $\tau=0$}

To perform the PRESTO experiment, we need for an irradiation power of at least $150 \mathrm{~W} / \mathrm{cm}$, in order to obtain a representative PWR decay heat for the fuel sample.

Our results show that the tungsten is a great absorber of thermal neutrons compared to the zirconium (see figure 5). As a result, we do not reach in the fuel sample, for each configuration, the required level of irradiation power; irradiation power is around $10-15 \mathrm{~W} / \mathrm{cm}$ in configurations 1 , 2 , and 4 , and $60 \mathrm{~W} / \mathrm{cm}$ in the configuration 3 .

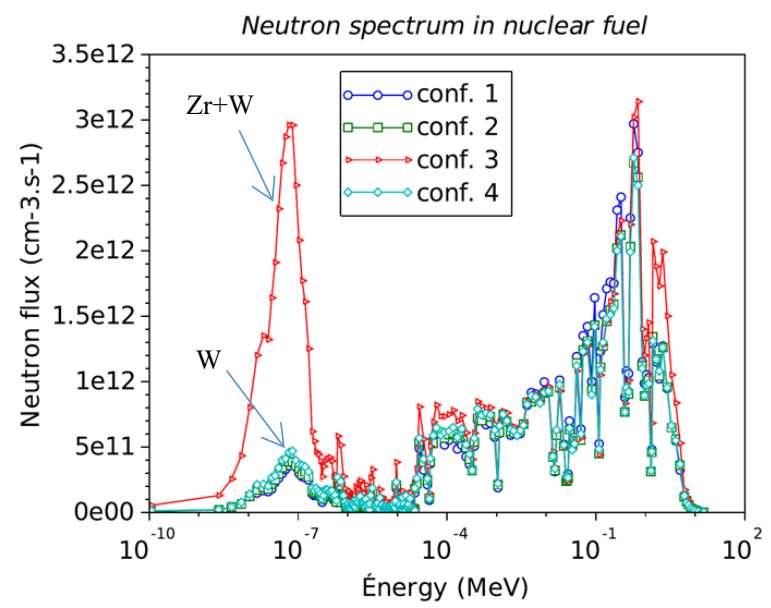

Fig. 5. The neutron spectrum in the fuel sample for each device configuration.

Thus, to achieve a PWR representative decay heat, we need to remove tungsten during the irradiation phase. This makes the experiment design more complicated, and solutions might correspond to design a shielding entirely made of zirconium, or provide vertical displacements for the tungsten shield.

Furthermore, irradiation duration is another important design parameter for the experiment representativity. We need simultaneously to reduce as much as possible the irradiation time, and to improve the experiment representativity.

\section{2) The measurement phase $\tau=2 \mathrm{~min}$}

During the measurement phase, two main power sources were considered to calculate nuclear heating: the decay heat of the fuel sample, and the decay heat of the reactor core. Our results show that they heat the device structures with a different azimuthal form factor. In particular, we found that fuel sample decay heat is released in device structures uniformly according to the azimuth, whereas reactor core decay heat is released mostly in the portion of device facing the reactor vessel (see figure 6).

Estimations were performed by defining, for each configuration, 8 azimuthal regions of equal volume (see figure 3). Such a result, it is important to study a discrimination method between the source to be measured and the perturbation, and improve the estimation of the inverse technique.

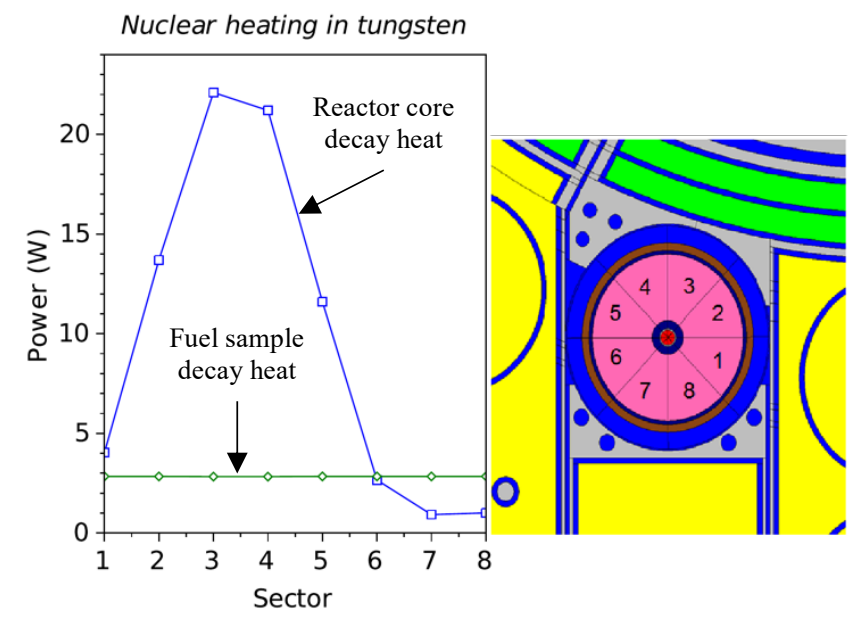

Fig. 6. Nuclear heating in the tungsten shield of the device configuration number 1. Fuel sample and reactor core contributions.

\section{CONCLUSION AND PERSPECTIVES}

Our results show that the TRIPOLI simulation is necessary to design the experimental device of the PRESTO experiment; in particular, in terms of experiment representativity and gamma shielding design. Furthermore, to maximize the measurement sensitivity, we have to match the neutronic simulation with the technological and metrological aspects of the PRESTO experiment.

\section{ACKNOWLEDGMENT}

I would like to thank David Blanchet (CEA, Cadarache), we benefit from his collaboration to perform the TRIPOLI study.

\section{REFERENCES}

[1] J-C. Benoit, "Développement d'un code de propagation des incertitudes des données nucléaires sur la puissance résiduelle dans les réacteurs à neutrons rapides", thèse de doctorat de l'université ParisSud (2012).

[2] N. Todreas, M. Kazimi, "Nuclear Systems Vol. 1”, CRC Press (2012).

[3] S. Bourganel, "Conception d'une experience de mesure de la puissance résiduelle d'un crayon irradié : l'expérience MERCI", thèse de doctorat de l'INPG (2002).

[4] F. Sturek, L. Agrenius, "Measurements of decay heat in spent nuclear fuel at the Swedish interim storage facility", Clab, Svensk Karnbranslehantering AB, Rapport R-05-62, (2006).

[5] C. Fiche, "Mesure de la puissance résiduelle totale émise par les produits de fission thermique de $239 \mathrm{Pu}$ et $233 \mathrm{U}$ ”, NEACRP/L(1978)212.

[6] F. Muratori, F. Nguyen, C. Gonnier, C. Le Niliot, R. Eschbach, "Modeling and sensitivity study of an experimental device for a decay heat measurement performed on an irradiated nuclear fuel sample in the very short cooling time range: the PRESTO experiment", Congrès Français de Thermique, 3-6 juin 2019, Nantes, France.

[7] J. Estrade, G. Bignan, X. Bravo, "The Jules Horowitz Reactor: a new high performance MTR working as an international user facility in support to nuclear industry, public bodies and research institutes", RRFM (2015).

[8] P.C. Hansen, "Rank deficient and ill-posed problems", siam (1998). 